\title{
Morphogenesis of Anthers Isolated From Cabbage (Brassica Oleracea L.) In Vitro
}

\author{
Rima Kirakosyan and Elena Kalashnikova \\ Russian Timiryazev State Agrarian University, Moscow, Russia \\ ORCID \\ Rima Kirakosyan; 0000-0002-5244-4311
}

Corresponding Author: Rima

Kirakosyan; email:

mia41291@mai.ru

\section{Dates}

Published 13 January 2022

Publishing services provided by Knowledge E

(c) Rima Kirakosyan and Elena Kalashnikova. This article is distributed under the terms of the Creative Commons

Attribution License, which permits unrestricted use and redistribution provided that the original author and source are credited.

Selection and Peer-review under the responsibility of the 8 th Scientific and Practical Conference Conference Committee.
Abstract. This study aimed to optimize the steps of obtaining regenerated cabbage plants by direct embryogenesis from isolated anthers and ovaries. Stepwise pretreatment of inflorescences was usedfor the studied hybrids and inbred lines. First, the inflorescences were placed in water and kept at a temperature of $+4-6^{\circ} \mathrm{C}$ for one day without the use of biologically active substances. Then the inflorescences were placed in a solution of the drug Dropp $(10 \mathrm{mg} / \mathrm{l})$ and cultivated for two days. After that, the anthers and ovaries were isolated from the flower buds and cultured on the MS culture medium at a temperature of $+32^{\circ} \mathrm{C}$ for one day. The cultivation of the isolated explants on a nutrient medium (containing $0.01 \mathrm{mg} /$ lof Dropp, $1.0 \mathrm{mg} / \mathrm{lof} \mathrm{NAA}, 500$ $\mathrm{mg} / \mathrm{lof}$ asparagine, $100 \mathrm{mg} / \mathrm{l}$ of tyrosine, and $10 \mathrm{~g} / \mathrm{l}$ of sucrose)led to an increase in their morphogenetic potential in the culture of anthers and ovaries (by 3.42\% and 5.54\%, respectively).A cytological method was usedto demonstrate the haploid nature of the regenerating plants. The number of chromosomes in the root meristem andleaves, and the chloroplasts in the closing cells of the stomatawere calculated.

Keywords: cabbage, culture in vitro, regenerated plants, anthers, ovaries, reproductive organs

\section{Introduction}

Currently, cabbage is the most common vegetable. The reason is its nice taste and chemical composition. It contains $2.6-5.7 \%$ of sugar, $1.1-2.3 \%$ of the proteins, minerals phosphorus, potassium, magnesium, zinc, manganese, iodine, etc. One of the greatest advantages of cabbage is its ability to maintain vitamin $C$ without reduction for 7-8 months. No vegetable has such kind of the property. In addition to vitamin $\mathrm{C}$, there are $\mathrm{B}_{1}, \mathrm{~B}_{2}, \mathrm{~B}_{6}, \mathrm{PP}$, carotene, folic acid. Among bioactive substances,tartronicacid, which holds the body turning carbohydrates into fat and thus prevents the accumulation of excess body weight, isof particular interest [1].

The most important problem of selection is still reducing the time to development of new varieties. For many years, the efforts of breeders have been mainly focused on the elimination of plant varieties of the intensive type, responsive to the widespread use of high doses of fertilizers, pesticides and favorable growing conditions. Required plant varieties are those combining high potential productivity with resistance to diseases and 
adverse environmental factors,for example, extreme temperatures, drought, salinity, soil acidity, lack of mineral nutrients, etc. New varieties should also have a good quality of products and the ability to withstand rapid changes in weather conditions. We address the complexity of such problems of traditional methods which requires a long time and a huge scope of work [2]. Broad prospects in the intensification of the selection process opens the application of modern techniques of applied genetics and applied biotechnology. These methods must be applied in combination with classical selection. These methods have practical and theoretical significance. Technologies for creating haploid plants are of great interest. In the early XX century, scientists were talking about the acceleration of the breeding process through the use of dihaploid plants [3]. Currently dihaploid plants are created through biotechnology. For this purpose, isolated anthers, microspores, ovaries, ovules, and embryos that lack one paternal chromosome are used. All primary explants are cultured on nutrient media in vitro [4,5], there wasthe cultivation of anthers and isolated microspores [6], unfertilized ovaries and ovules [ 7-9].

The method of anther culture and isolated microspore is one of the most promising ways to obtain haploid plants in vitro. The culture of microspores and pollen is a model system for studying the processes of plant regeneration from single-celled systems. In addition, the correct selection of conditions for the cultivation of anthers and microspores that ensure the formation of somatic embryos is the subject of biochemical, morphological and molecular studies of the mechanisms of embryogenesis. Thus, the doubled haploid plants can be successfully used in selecting mutants of cellular structures. Efficiency of mutagenesis at the haploid level is obvious as for diploid cells on the same locus double mutationis required, but we would rather focus on haploid mutation in a single locus, followed by a doubling of the chromosomes.

The results showed that there is still embryogenesis in anther culture in vitro of different Brassica species, which occurs spontaneously and has a low output frequency of haploid plants (1-4\%). The proposed technologies are difficult to reproduce and are not sufficiently studied at every stage of androgenesis. Therefore, optimization of conditions for cultivation of reproductive organs in vitro is an urgent problem important for breeding.

\section{Methods and Equipment}

The work was carried out on breeding the samples of white cabbage (Brassica oleracea L.): Zimovka 1474, SlavaGribovskaya 231, as well as the breeding material (inbred lines - 45-1, 112-1, 11-30 and breeding forms). The material was obtained in the Research Institute of Selection and Seed Production of Vegetable Crops (VNIISSOK) (Moscow 
region). Plants - donors - were grown in artificial climate chambers of the Federal Scientific Center for Vegetable Production.

Objects of our research were isolated anthers, ovaries and ovules of cabbage. First, the inflorescences are placed in water and kept at a temperature $\left(+4-6^{\circ} \mathrm{C}\right)$ for 1 day without the use of biologically active substances. Then the inflorescences were placed in a solution of the drug Dropp ( $10 \mathrm{mg} / \mathrm{l})$ and cultivated for 2 days. After that, the anthers and ovaries were isolated from flower buds and cultured on MS culture medium at a temperature of $+32^{\circ} \mathrm{C}$ for 1 day.

For surface sterilization of primary explants we used a sterilizing agent mercuric chloride $\left(\mathrm{HgCl}_{2}\right)$ at $0.1 \%$. Isolated buds were kept in mercuric chloride for 4 - 5 minutes, after which they were washed in three portions of sterile distilled water.

In culture media we used by the prescription Murashige and Skoog (MS) with added $2 \%$ of sucrose, $0.8 \%$ of agar, $500 \mathrm{mg} / \mathrm{l}$ of asparagine, $100 \mathrm{mg} / \mathrm{l}$ of tyrosine and plant hormones. There were substances withcytokinin activity using Dropp at concentrations of $0.01 \mathrm{mg} / \mathrm{l}$, substances such as auxin - naphthylacetic acid (NAA) concentrations of 1.0 $\mathrm{mg} / \mathrm{l}$. Control variant-MS culture medium with 6-benzylaminopurine (BAP) of $1 \mathrm{mg} / \mathrm{l}$ was in combination with NAA $-0.5 \mathrm{mg} / \mathrm{l}$. pH culture media in all variants was in the range of 5.5-5.8.

Anthers were aseptically removed from the buds in a laminar box and cultured on MS-inducing culture media in petri dishes were placed in climatic chamber (Binder, Germany) and incubated in the dark at $35^{\circ} \mathrm{C}$ for 24 hours, after which the temperature was reduced to $25^{\circ} \mathrm{C}$. In these conditions the explants cultured for 25 days and then transferred to light a room, which set a 16-hour photoperiod and light white fluorescent light with an intensity of 5 thousand lux.

The paper stuck to the rules work in a sterile environment, developed at the Department of Biotechnology RSAU-MAA named after K.A.Timiryazev [10].

Cytological analysis of counting the quantity of chromosomes and chloroplasts in stomatal guard cells was carried out in accordance with the workshop on cytology and cytogenetics of plants [11].

\section{Results}

The success of obtaining callus and / or embryogenic haploid structures depends on the correct estimation of the initial state of the gametophyte of the donor plant. We study the dependence of the stage of development of microspores from the bud size and its location in the main and lateral inflorescences (Table 1). 
TABLE 1: The content of microspores at different stages of development in the anther, depending on the location of the buds in the inflorescence of cabbage (B. oleracea L.),\%

\begin{tabular}{|c|c|c|c|}
\hline \multirow[t]{2}{*}{$\begin{array}{l}\text { Row of buds } \\
\text { center }\end{array}$} & \multicolumn{3}{|c|}{ Several stages of microspore } \\
\hline & $1-2$ & $3-6$ & $7-9$ \\
\hline center florets & $79.9(77.2 \div 83.0)^{*}$ & $5.7(1.4 \div 6.7)$ & 0 \\
\hline $1-2$ the row & $24.2(16.1 \div 29.3)$ & $\begin{array}{l}76.3 \\
(67.2 \div 83.0)\end{array}$ & 0 \\
\hline $3-4$ the row & $11.1(5.7 \div 15.2)$ & $88.1(82.1 \div 91.2)$ & 0 \\
\hline 5-6the row & 0 & $10.9(6.3 \div 16.4)$ & $81.3(75.1 \div 87.1)$ \\
\hline$>6$ & 0 & 0 & $98.9(95.4 \div 99.9)$ \\
\hline
\end{tabular}

As can be seen from table 1, in buds located in 1-4 rows from its center, microspores are in a single-core stage of development. This pattern can be seen in the buds located both in the main and lateral inflorescences. The content of microspores in anthers varied at an early stage from high mononuclear (stage 3) to late mononuclear (stage 6) was 76.3-88.1\%. In addition, when selecting buds, you must take into account the color of the buds. This color should be light green. In buds isolated from 5 or more rows, the formation of pollen in the anthers is observed. Such anthers are not capable of plant regeneration. Therefore, to obtain haploid plants in vitro, it is necessary to isolate the buds located in rows 1-4 from the center.

It is known that growth regulators are a wide range of natural and synthetic substances that affect the growth and development of plants [12].Among them there are natural hormones and their synthetic analogues. These hormones are regulatory factors of plant physiological processes. Therefore, they can be used in plant biotechnology in vitro.

Our research has shown that the most successful combination of hormones in the culture medium for the cultivation of reproductive organs of $B$. oleracea $L$. in vitro is the combination of Dropp at a concentration of $0.01 \mathrm{mg} / \mathrm{l}$ with NAA-1.0 mg/l [13]. Under these conditions of cultivation, the formation of callus tissue was observed. The effectiveness of callus formation for isolated anthers and ovaries was 1.5 times higher than the control variant (BAP $1 \mathrm{mg} / \mathrm{l}$ in combination with NAA $-0.5 \mathrm{mg} / \mathrm{l}$ ) and amounted to $17.8 \%$ and $29.6 \%$, respectively. The callus tissue was formed yellow and had a nonuniform structure. In the process of transplanting to a new nutrient medium, the callus tissue acquired a denser structure and became green in color. In this callus tissue, roots and somatic embryogenesis were formed. In the future, regenerating plants were formed from the somatic embryogenesis (Fig. 1). 


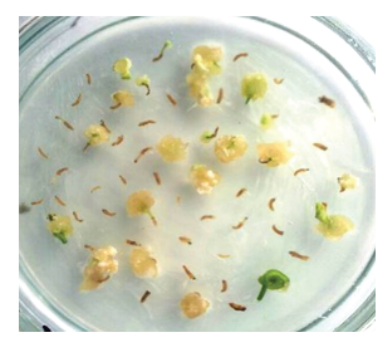

a

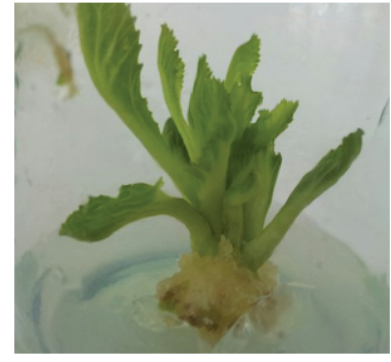

d

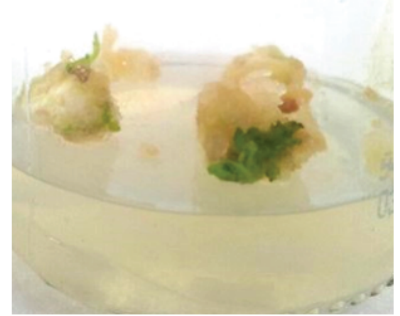

b

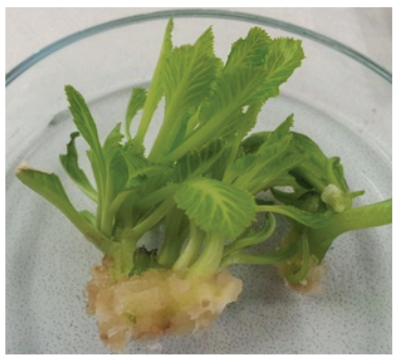

f

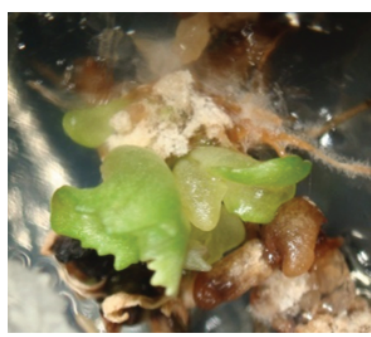

c

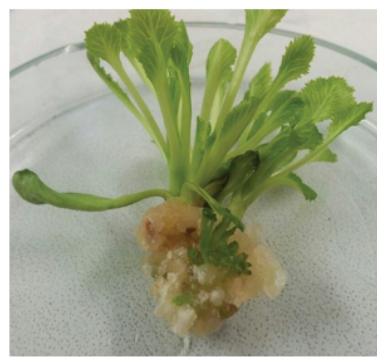

g

Figure 1: The formation of callus tissue (a); formation of meristematic zones (b); formation of somatic embryogenesis (c); plants from inbred lines 45-1 (d); plants from inbred lines 112-1 (f); plants frominbred lines $11-30(\mathrm{~g})$.

Subsequent culturing of callus tissue on MS culture medium with Dropp and NAA induced the formation of adventitious kidneys. The frequency of their formation in callus tissue obtained from anthers was $1.85 \%$, and from ovaries $-3.7 \%$. When replacing NAA with 2.4-D, the intensity of callus formation increased. In the culture of anthers, the frequency of callus formation was $16.7 \%$, and in the culture of ovaries-27.8\%. Under these conditions, the frequency of organogenesis was $1.11 \%$ and $1.85 \%$, respectively.

The optimized nutrient medium was also tested on other breeding samples provided by VNIISSOK employees. Studies have shown that the competence of anthers and ovaries to morphogenesis in vitro depends on the genotype. For example, for varieties Zimovka 1474, SlavaGribovskaya 231, the frequency of organogenesis was $0.8-1 \%$, and for inbred lines - 45-1, 112-1, 11-30, the frequency of organogenesis was 1.8-3.5\%.

Differentiated adventitious buds during culture evolved microshoots that showed strong growth, the formation of the correct morphology leaf and root system. For more active development of the root system, the nutrient medium should include auxins in higher concentrations (1 mg/I NAA).

Formed regenerated plants were subsequently transferred to the soil conditions of cultivation. The positive influence of the peat-turf substrate (1:1) adapts the regenerated plants of cabbage to the conditions ex vitro (Fig. 2).

The obtained regenerated plants were used to calculate the number of chromosomes in the root meristem, as well as the number of chloroplasts in the cells of the closing stomata of leaves using the new universal method of preparing preparations of plant 


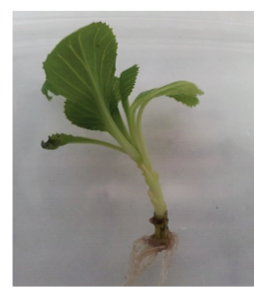

a

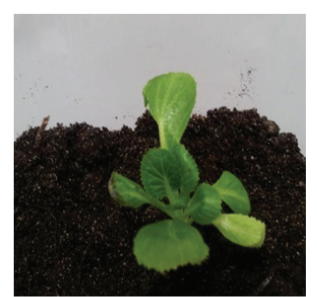

b

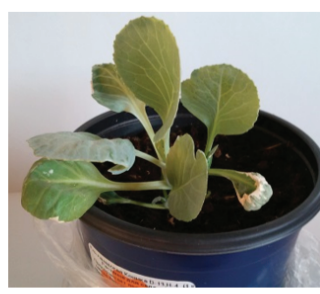

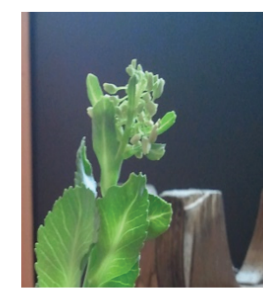

$\mathrm{d}$

Figure 2: Adaptation of white cabbage regenerated plants ex vitro: $a$ - microshoots with roots; $b$ - plants ex vitro after 3 months of adaptation, c - plants ex vitro after 4 months of adaptation; $d$ - formation of reproductive organs on plants.

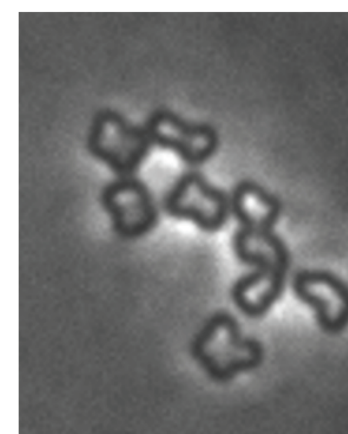

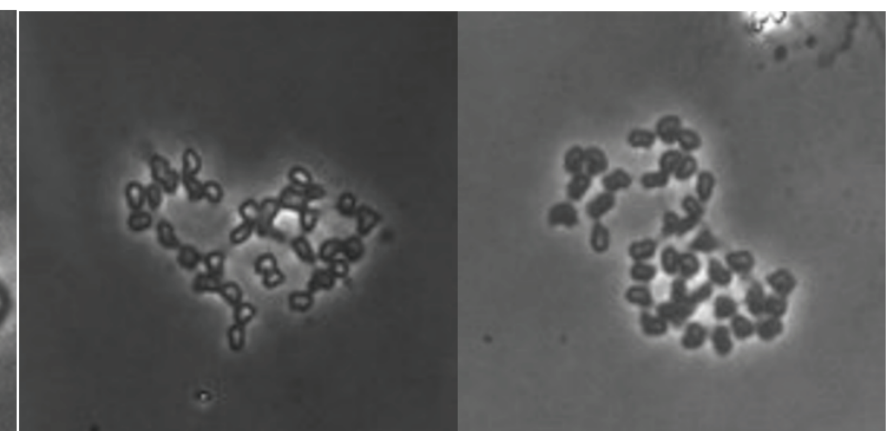

$3 n$ 5n

Figure 3: Cytological analysis of the chromosome set of white cabbage regenerated plants obtained from the reproductive organs (single $(n=9)$, triploid $(3 n=27)$ and pentaploid $(5 n=45)$ ).

chromosomes - «SteamDrop» [15]. As a result of the research, the dependence of the level of ploidy on the cultivation conditions was studied. It has been shown that plants-regenerants of white cabbage, obtained in vitro from reproductive organs, had a different set of chromosomes ( $n, 3 n, 5 n)(F i g .3)$. It was established that the number of chloroplasts in the stomatal cells of regenerated plants was from 9 to 11 , while the original donor plants had 18-20.

\section{Discussion}

One of the most important goals of plant breeding is creating $\mathrm{DH}$ lines. It allows you to reduce the time of selection and get new varieties. Much attention is paid to in vitro haploid technology worldwide. However, these technologies have their drawbacks, because the frequency of haploid plant regeneration from anthers and microspores is low $[4,6-8,15]$. Therefore, research is underway to improve these technologies. The use of drugs with cytokinin activity increases the frequency of plant regeneration in vitro culture. This work presents a non-standard technology for pre-treatment of buds before their introduction in vitro. In addition, the drug Droppsignificantly increases the morphogenetic potential of isolated anthers and microspores. The drug Dropp was 
used in the treatment of inflorescences before their introduction in vitro. The technology provides for placing inflorescences in a solution of the drug Drop $(10 \mathrm{mg} / \mathrm{l})$ for 2 days. After that, the anthers and ovaries were isolated from flower buds and cultured on MS culture mediumwithcombination of Dropp at a concentration of $0.01 \mathrm{mg} / \mathrm{l}$ with NAA-1.0 $\mathrm{mg} / \mathrm{I}$.This method has not been used in technology to create $\mathrm{DH}$ lines before. One of the most important aspects of this technology is the proof of the haploid nature of regenerating plants.The method "SteamDrop" [14]that was used in this work was developed in the Russian state agrarian University - MSHA and tested on different plants.

\section{Conclusion}

Thus, on the basis of the experimental data, we picked up the culture conditions that would ensure the regenerated plants of cabbage isolated reproductive organs and proved their haploid nature.

\section{Acknowledgement}

The authors would like to thank their colleague for their contribution and support to the research. They are also thankful to all the reviewers who gave their valuable inputs to the manuscript and helped in completing the paper.

\section{Conflict of Interest}

The authors have no conflict of interest to declare.

\section{References}

[1] Petrushko YN.White cabbage. Modern resource-saving growing technology in the Primorsky Territory.Ussuriysk:AcademicPublishers; 2003.

[2] Bondareva LL, PivovarovVF.The main directions and results of selection and seed production of cabbage crops in VNIISSOK. Journalof Vegetables of Russia. 2013;3:49.

[3] MayDC. Improving the technology for haploid and digaploid rape plants (Brassica napus I.) andcabbage (Brassica oleracea I.) in vitro[PhD dissertation]. Moscow, Russian Timiryazev State Agrarian University; 2010. 
[4] Gil-Humanes J, Barro F. Production of doubled haploids in Brassica. Advances in haploid production in higher plants. Luxembourg, Springer, Biomedical and Life Sciences; 2009.

[5] Akmal M, Nafis T, Mirza KJ et al. High frequency somatic embryogenesis in mustard crop (Brassica juncea L. cv. Pusa Jai kisan): Microscopic and histological analyses. Australian Journal of Crop Science. 2011;5(13):1783- 1789.

[6] Na H, Hwang G, Kwak JH, Yoon MK, Chun C. Microspore derived embryo formation and doubled haploid plant production in broccoli (Brassica oleracea L. varitalica) according to nutritional and environmental conditions. African Journal of Biotechnology.2011;10(59):12535-12541.

[7] Pink D, Bailey L, McClement S et al. Double haploids, markers and QTL analysis in vegetable Brassicas. Euphytica.2008;164:509-514.

[8] Tuncer B, Ruhsar Y. Induction of microspore embryogenesis in ornamental kale by gamma irradiation and high temperature stress. Asian Journal of Biotechnology.2011;3:415-421.

[9] JongIP, Nasar UA, Hye-Ran K. (2012). Advances in in vitro culture of the Brassicaceae crop plants. Plant Biotechnology Journal. 2012;39:13-22.

[10] KalashnikovaEA, Cherednichenko MY, Kirakosyan RN, Zaitseva SM. (2017). Laboratory workshop on the culture of cells and plant tissues. Moscow: FGNU "Rosinformagrotex"; 2017.

[11] Puchalski VA, Soloviev AA, Badaeva ED, Yrtsev VN Workshop on cytology and cytogenetics of plants. Moscow: Colossus; 2007.

[12] Muromtsev GS, Butenko RG, Tikhonenko TI, Prokofiev MI.The basics agricultural biotechnology. Moscow: Agropromizdat; 1990.

[13] Kirakosyan RN, Kalashnikova EA. (2016). Cytological and biochemical analysis of plants-regenerators of white cabbage (Brassica oleracea L.) obtained from in vitro reproductive organs. Presented at: VII International Scientific and Practical Conference dedicated to the 30th anniversary of the Department of Plant Biotechnology Nikitsky Botanical Garden; September 25th-October 1st, 2016, Yalta (Russia).

[14] Kirov I, Divashuk M, Van LaereK et al. An easy "SteamDrop" method for high quality plant chromosome preparation. Molecular Cytogenetics. 2014;7:21-21.

[15] Jahangir M, Kim HK,Choi YH, Verpoorte R. Health-affecting compounds in Brassicaceae. Comprehensive Reviews in Food Science and Food Safety. 2009;8:31-43. 